\title{
Appropriate Needle Lengths Determined Using Ultrasonic Echograms for Intramuscular Injections in Japanese Infants
}

\author{
Tetsuo Nakayama ${ }^{*}$, Urara Kohdera ${ }^{2}$, Motoko Fujino ${ }^{3}$, Toshihiro Tanaka4, Keitaro Yatabe ${ }^{5}$, \\ Tomoyuki Hashiguchi ${ }^{5}$, Tomohide Sato ${ }^{5}$, Minoru Kino ${ }^{2}$ \\ ${ }^{1}$ Kitasato Institute for Life Sciences, Laboratory of Viral Infection I, Tokyo, Japan \\ ${ }^{2}$ Department of Pediatrics, Nakano Children's Hospital, Osaka, Japan \\ ${ }^{3}$ Department of Pediatrics, Saiseikai Central Hospital, Tokyo, Japan \\ ${ }^{4}$ Department of Pediatrics, Shizuoka Kosei Hospital, Shizuoka, Japan \\ ${ }^{5}$ Department of Clinical Development \& Medical Affairs, Japan Vaccine Co., Ltd., Tokyo, Japan \\ Email: tetsuo-n@lisci.kitasato-u.ac.jp
}

Received 27 April 2016; accepted 3 June 2016; published 6 June 2016

Copyright $@ 2016$ by authors and Scientific Research Publishing Inc.

This work is licensed under the Creative Commons Attribution International License (CC BY). http://creativecommons.org/licenses/by/4.0/

(c) () Open Access

\section{Abstract}

Adjuvanted vaccines are recommended for administration through an intramuscular route. The Centers for Disease Control and Prevention (CDC) has recommended the anterolateral thigh using a 22 - 25-G $25-\mathrm{mm}(1 \mathrm{inch})$ needle for infants, injected at a $90^{\circ}$ into the skin surface, and using a 16 mm (5/8 inch) for newborns. Appropriate needle lengths may differ depending on racial backgrounds. In the present study, the thickness of the epidermis and lengths from the skin surface to the muscle fascia and bone were measured using ultrasonic echograms in order to determine suitable needle lengths for Japanese infants aged 2, 3, 4, 5, 6, and 12 - 15 months old. The thickness of the epidermis was $1.44-1.54 \mathrm{~mm}(95 \% \mathrm{CI})$, and the lengths from the skin surface to the muscle fascia and bone were $11.52-12.28 \mathrm{~mm}(95 \% \mathrm{CI})$, and $25.66-26.93 \mathrm{~mm}(95 \% \mathrm{CI})$, respectively, at the anterolateral thigh. At the center of the deltoid muscle, skin thickness was similar to that at the thigh, furthermore the lengths from the skin surface to the muscle fascia and bone were 8.49 $9.10 \mathrm{~mm}(95 \% \mathrm{CI})$, and $17.38-18.31 \mathrm{~mm}(95 \% \mathrm{CI})$, respectively. The lengths from the skin surface to the muscle fascia and bone were $1-2 \mathrm{~mm}$ shorter in 2-month-old infants than those in older generations. Therefore, the appropriate needle length for intramuscular injections in Japanese infants was $16 \mathrm{~mm}(5 / 8 \mathrm{inch})$ at any age and sites, and with $25 \mathrm{~mm}(1 \mathrm{inch})$ needles at a $90^{\circ}$ angle being associated with the risk of over-penetration.

\section{Keywords}

Intramuscular Injection, Subcutaneous Injection, Vaccines, Needle Length

\footnotetext{
${ }^{*}$ Corresponding author.
}

How to cite this paper: Nakayama, T., Kohdera, U., Fujino, M., Tanaka, T., Yatabe, K., Hashiguchi, T., Sato, T. and Kino, M. (2016) Appropriate Needle Lengths Determined Using Ultrasonic Echograms for Intramuscular Injections in Japanese Infants. Open Journal of Pediatrics, 6, 163-170. http://dx.doi.org/10.4236/ojped.2016.62024 


\section{Introduction}

All vaccines have been administered using Subcutaneous Injections (SC) since 1970 in Japan, and Intramuscular Injections (IM) being prohibited, due to muscle contracture of the quadriceps caused by the repeated administration of antibiotics and antipyretics through IM [1]. The first case was reported in 1946 and several cases were subsequently reported by the Orthopedic Medical Association between 1960s and 1970s. Some orthopedic medical doctors strongly recommended general physicians to refrain from IM. A number of young infants and children with delayed development in sitting and walking or awkward walking postures were reported in Yamanashi prefecture 1973. This was diagnosed as contracture of the femur quadriceps muscle and resulted in low suits. The Japan Pediatric Society proposed that muscle contracture was caused by the repeated administration of antibiotics and antipyretics through IM. There was no safety site and generation, and IM administration should be avoided. Thereafter, the administration of all medicinal products by IM was prohibited even though no case was reported following vaccinations. All vaccines were subsequently administered SC [1].

Some pharmaceutical products are currently administered IM such as immunoglobulin for prophylaxis against measles or the treatment of some infectious diseases and palivizumab. All adjuvanted vaccines are administered IM worldwide [2]. Recently, 10-valent Pneumococcal conjugate vaccine was regulatory approved as IM injection, and HBV vaccine which can be administered by SC or IM is adopted for National Immunization Program (NIP) in 2016 in Japan. However, other vaccines are still administered by SC. Histopathological findings revealed the presence of inflammatory nodules following the administration of alum-adjuvanted vaccines, but not non-adjuvanted vaccines. Furthermore, no significant differences were observed in histopathological findings between IM and SC [3].

IM has several advantages including a lower incidence of local adverse events and greater immunogenicity [4] [5]. IM is a safe and effective procedure that needs to be more strongly recommended. In Japan, SC has been conventionally employed by general practitioners. Although guidelines exist for appropriate sites, procedures, and needle sizes [4] [5], needle lengths may differ in Japanese infants. In the present study, the lengths from the skin surface to the muscle fascia and bone were measured to determine the appropriate lengths of vaccination needles for IM in Japanese infants using ultrasonic echograms.

\section{Materials and Methods}

\subsection{Study Design}

In order to assess needle lengths for IM, 184 healthy Japanese infants were enrolled from those who visited the Department of Pediatrics of three general hospitals from $14^{\text {th }}$ August to $17^{\text {th }}$ November 2015: Saiseikai Central Hospital, Tokyo, Shizuoka Kosei Hospital, Shizuoka, and Nakano Children's Hospital, Osaka. 31 infants aged two months, 33 aged three months, 30 aged four months, 30 aged five months, 30 aged six months, 30 aged 12 months or older but less than 15 months (12 - 15 months) were enrolled, and totally male and female were 94 and 90, respectively. Target months of age were determined according to the recommended immunization schedule for infants by the Japan Pediatric Society. Body weight, height, and the circumferences of the upper arm at the center of the deltoid muscle, and the lower $1 / 3$ of the upper arm, and the middle position of thigh were measured. Inclusion and exclusion criteria were as follows:

Inclusion criteria

1. Written consent was obtained from infants' family.

2. Infants who has grown healthy after birth without any chronic ailments. Even if infants had some chronic illness, they were allowed when medical investigators judged as no influence on the measurement.

Exclusion criteria

1. Infants who had an abnormality in their extremities.

2. Infants with serious chronic ailments relating developmental disorders, neurological or muscular diseases.

3. Infants not cooperative in measurement.

4. Infants whose written consent was not obtained by their family.

Subject backgrounds were shown in Table 1 . Height and body weight increased in an age-dependent manner. Most infants had gestational periods of 37 - 42 weeks and BMI of 16 - 18.

The thickness of the epidermis and lengths from the surface of the skin to the muscle fascia and bone were measured using ultrasonic echograms. The objectives and the design of the present study were explained to infants' family (guardians or legally acceptable representatives), and written informed consent was obtained by 
Table 1. Subject backgrounds.

\begin{tabular}{|c|c|c|c|c|c|c|c|}
\hline & & $2 \mathrm{M}$ & $3 \mathrm{M}$ & $4 \mathrm{M}$ & $5 \mathrm{M}$ & $6 \mathrm{M}$ & $12-15 \mathrm{M}$ \\
\hline \multicolumn{2}{|c|}{ Number of subjects } & 31 & 33 & 30 & 30 & 30 & 30 \\
\hline \multirow{6}{*}{ Height } & $<55 \mathrm{~cm}$ & 1 & 0 & 1 & 0 & 0 & 0 \\
\hline & $55-<60 \mathrm{~cm}$ & 27 & 10 & 1 & 0 & 0 & 0 \\
\hline & $60-<65 \mathrm{~cm}$ & 3 & 20 & 21 & 21 & 9 & 0 \\
\hline & $65-<70 \mathrm{~cm}$ & 0 & 2 & 6 & 8 & 19 & 4 \\
\hline & $70-<75 \mathrm{~cm}$ & 0 & 0 & 1 & 0 & 2 & 14 \\
\hline & $75-<80 \mathrm{~cm}$ & 0 & 0 & 0 & 0 & 0 & 11 \\
\hline \multirow{9}{*}{$\begin{array}{c}\text { Body } \\
\text { weight }\end{array}$} & $\geq 80 \mathrm{~cm}$ & 0 & 0 & 0 & 0 & 0 & 1 \\
\hline & $<5000 \mathrm{~g}$ & 3 & 0 & 2 & 0 & 0 & 0 \\
\hline & $5000-<6000 \mathrm{~g}$ & 20 & 9 & 0 & 3 & 0 & 0 \\
\hline & $6000-<7000 \mathrm{~g}$ & 6 & 18 & 17 & 9 & 7 & 0 \\
\hline & $7000-<8000 \mathrm{~g}$ & 2 & 3 & 9 & 12 & 15 & 4 \\
\hline & $8000-<9000 \mathrm{~g}$ & 0 & 2 & 2 & 4 & 7 & 12 \\
\hline & $9000-<10000 \mathrm{~g}$ & 0 & 0 & 0 & 1 & 1 & 10 \\
\hline & $10000-<11000 \mathrm{~g}$ & 0 & 0 & 0 & 0 & 0 & 4 \\
\hline & $\geq 11000 \mathrm{~g}$ & 0 & 0 & 0 & 0 & 0 & 0 \\
\hline \multirow{5}{*}{$\begin{array}{l}\text { Gestational } \\
\text { period }\end{array}$} & $<27 \mathrm{~W}$ & 0 & 0 & 0 & 0 & 0 & 0 \\
\hline & $27-31 \mathrm{~W}$ & 0 & 0 & 0 & 0 & 0 & 0 \\
\hline & 31 - 37 W & 1 & 0 & 2 & 2 & 1 & 6 \\
\hline & $37-42 \mathrm{~W}$ & 30 & 33 & 28 & 28 & 29 & 24 \\
\hline & $\geq 42 \mathrm{~W}$ & 0 & 0 & 0 & 0 & 0 & 0 \\
\hline \multirow{5}{*}{ BMI } & $<14$ & 0 & 0 & 1 & 0 & 0 & 1 \\
\hline & $14-<16$ & 9 & 9 & 8 & 5 & 7 & 12 \\
\hline & $16-<18$ & 16 & 17 & 14 & 20 & 13 & 16 \\
\hline & $18-<20$ & 6 & 5 & 6 & 3 & 10 & 1 \\
\hline & $\geq 20$ & 0 & 1 & 1 & 1 & 0 & 0 \\
\hline
\end{tabular}

infants' family. This study protocol was reviewed and approved by the Ethics Committees of Saiseikai Central Hospital, Shizuoka Kosei Hospital, and Nakano Children's Hospital. This study was conducted in accordance with Ethical Guidelines for Medical and Health Research Involving Human Subjects (Dec, 2014 The Ministry of Education, Culture, Sports, Science and Technology; Ministry of Health, Labour and Welfare) and ethical Principles based on Declaration of Helsinki. Colored cells represent the majority of each category.

\subsection{Ultrasonic Echogram}

Ultrasonic echograms were performed on the middle of the deltoid muscle and centro-lateral thigh using Viamo SSA-640A with the linear probe PLT-740AT (Toshiba Medical Systems, Japan), Aplio 400/500 with the linear probe PLT-704SBT (Toshiba Medical Systems, Japan), and Prosound SSD- $\alpha 10$ with the linear probe UST-5411 (Hitachi Aloka-Medical, Japan). The thickness of the epidermis, and the lengths from the skin surface to the muscle fascia and bone were measured based on the different intensities of echograms, with stretching the skin flat.

\subsection{Statistical Analysis}

190 subjects were enrolled into the present study and 184 subjects were analyzed. 6 subjects were excluded from analysis because those informed consent forms were not re-obtained after its version was updated. Ultrasonic 
images were examined by a medical echogram specialist and unclear blurred images were excluded from the analysis. Statistical analyses were performed using SAS ver. 9.2 software.

\section{Results}

\subsection{Circumferences}

The circumferences of the thigh, deltoid muscle, and lower $1 / 3$ of the upper arm were measured and the results obtained are shown in Figure 1. The circumferences of the thigh increased until four months of age. The circumferences of the upper arm around the deltoid muscle and the lower 1/3 were similar from two months to 12 15 months of age.

\subsection{Lengths from the Surface of the Skin to the Muscle Fascia and Bone at the Thigh}

An ultrasonic image from a five-months of age male is shown in Figure 2, and the thickness of the epidermis, subcutaneous tissues, muscle fascia, and bone were identified through different ultrasonic intensities, for example, in the right panel of Figure 2. The thickness of the epidermis and lengths from the skin surface to the muscle fascia and bone in the different age groups are shown with 95\% CI. The thickness of the epidermis ranged between 1.44 and $1.54 \mathrm{~mm}$ with no differences being observed among the different age groups. The length from the skin surface to the muscle fascia was 9.39 - $10.86 \mathrm{~mm}$ in infants aged two months and increased to 12.14 $14.20 \mathrm{~mm}$ in those aged five months. The length from the skin surface to the bone was $22.35-25.06 \mathrm{~mm}$ in those aged two months, and increased to $26.00-29.05 \mathrm{~mm}$ in those aged five months. Dotted lines showed 5/8 inch (16-mm) and 1 inch (25-mm). The 16-mm needle penetrated to the muscle in all age groups, while the 25$\mathrm{mm}$ needle reached the bone in infants aged two months.

\subsection{Lengths from the Surface of the Skin to the Muscle Fascia and Bone at the Deltoid Muscle}

An ultrasonic image shown in the right panel of Figure 3 was taken from the same infant in Figure 2, and the thickness of the epidermis, subcutaneous tissue, muscle fascia, and bone were identified through different ultrasonic intensities. The thickness of the epidermis at two months of age was $1.57-1.89 \mathrm{~mm}$, thickest among the different age groups. The length from the skin surface to the muscle fascia was $8.39-9.74 \mathrm{~mm}$ in those aged two months, remained at a similar length until five months of age, and then decreased after six months of age. The length from the skin surface to the bone was $17.38-18.31 \mathrm{~mm}$ in all age populations tested.

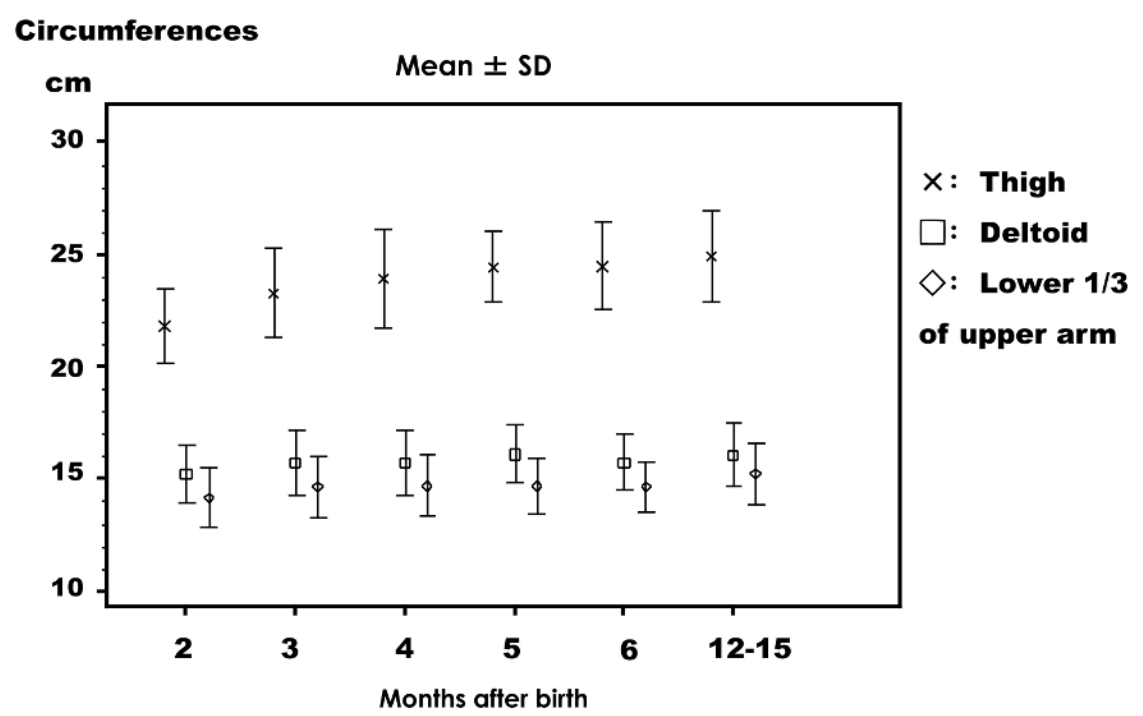

Figure1. Circumferences of the thigh, upper arm at the deltoid muscle, and lower $1 / 3$ of the upper arm in the different age groups (months). Circumferences are shown as the mean $\pm \mathrm{SD}(\mathrm{cm})$. 


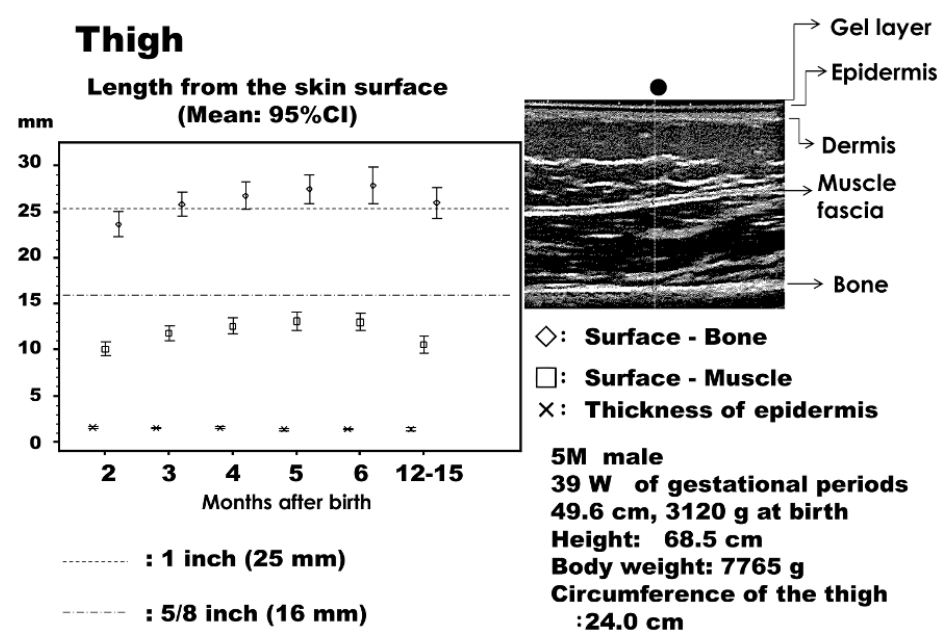

Figure2. Thickness of the epidermis and lengths from the skin surface to the muscle fascia and bone in the thigh. Length is shown as the mean with $95 \% \mathrm{CI}(\mathrm{mm})$ and the echogram is shown with a mark indicating the injection site.

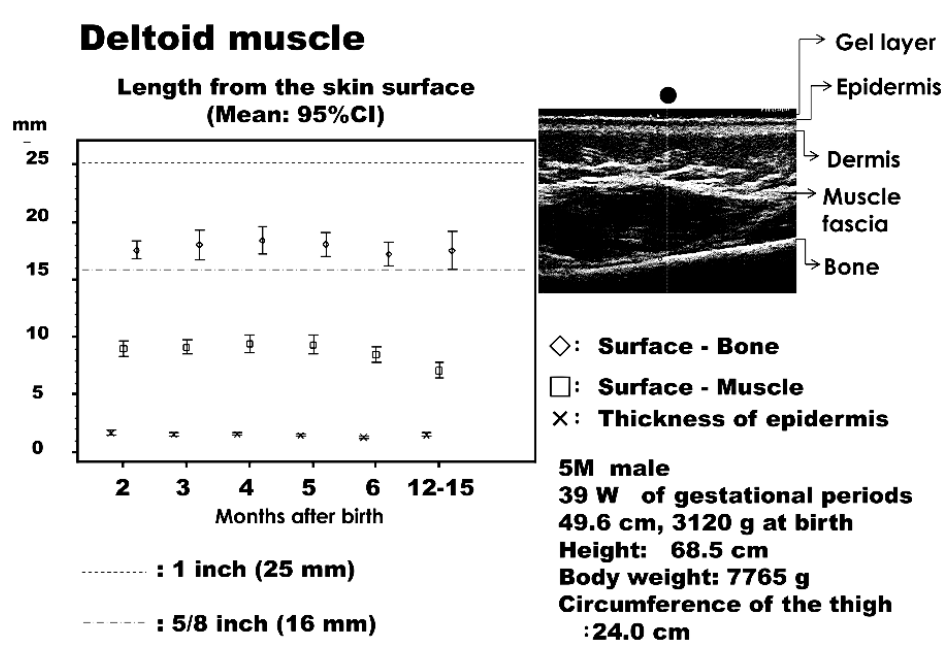

Figure 3. Thickness of the epidermis and the lengths from the skin surface to the muscle fascia and bone at the deltoid muscle. Length is shown as the mean with $95 \% \mathrm{CI}(\mathrm{mm})$ and an echogram is shown with a mark indicating the injection site.

\subsection{Lengths from the Surface of the Skin to the Muscle Fascia and Bone in the Lower $1 / 3$ of the Lateral Upper Arm}

SC is recommended for the lower $1 / 3$ of the lateral upper arm, whereas IM is not because of the small muscle volume. The thickness of the epidermis was $1.26-1.35 \mathrm{~mm}$, in all age populations tested, which was less than that at the other sites. However, the lengths from the skin surface to the muscle fascia and bone were similar to those at the deltoid muscle (Figure 4).

Data obtained in the different age groups are shown in Figure 5. Based on the results obtained, an appropriate needle length was considered to be $5 / 8$ inch $(16 \mathrm{~mm})$, injected at a $90^{\circ}$ angle into the skin surface, for any site and age.

\section{Discussion}

A popular injection technique is bunching the thigh muscle at the injection site in order to increase the muscle 


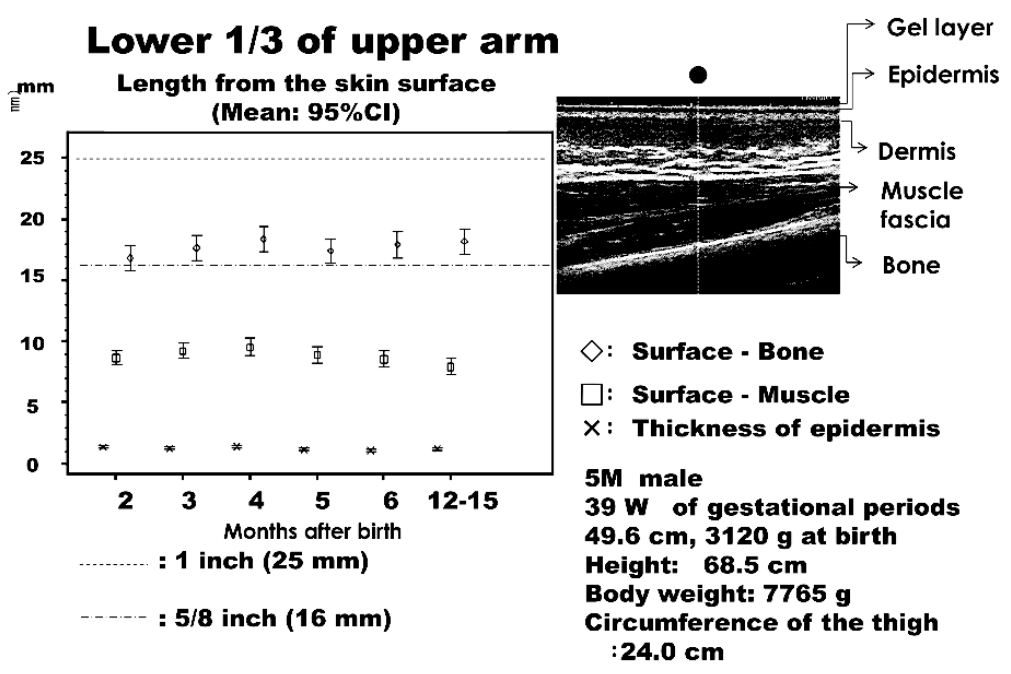

Figure 4. Thickness of the epidermis and lengths from the skin surface to the muscle fascia and bone at the lower $1 / 3$ of the upper arm. Length is shown as the mean with $95 \%$ CI (mm) and an echogram is shown with a mark indicating the injection site.

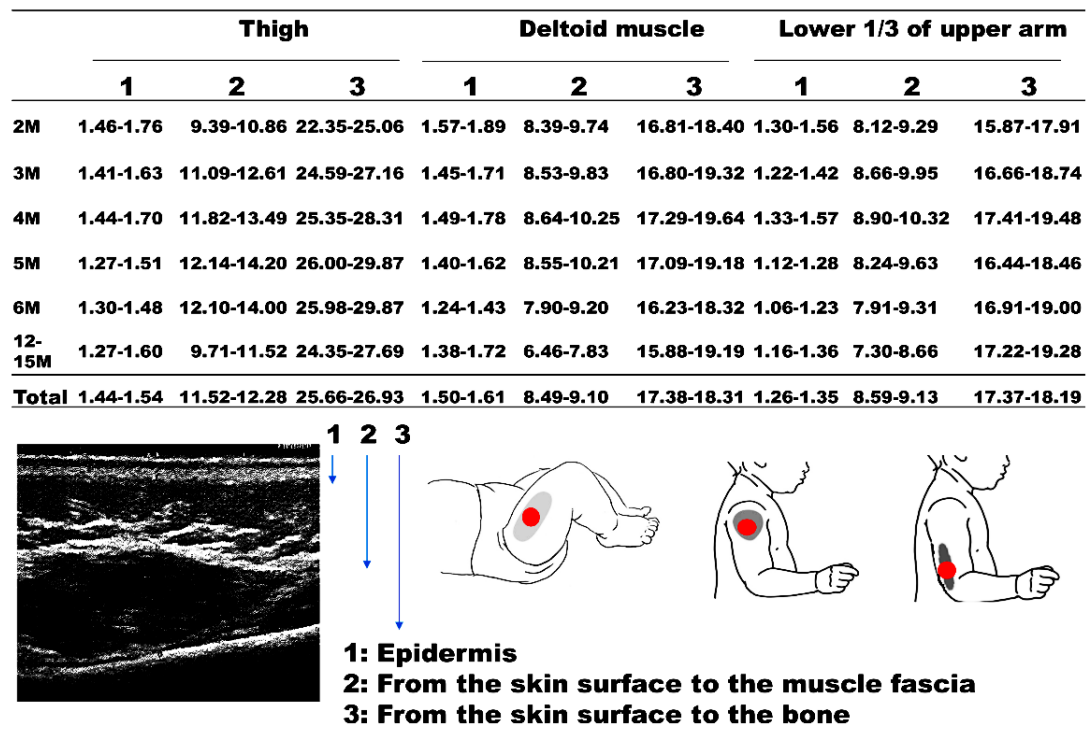

Figure 5. Summary of lengths at injection sites in different age groups (2, 3, 4, 5, 6, and 12 - 15 months of age), shown as 95\% CI (mm). Red marks represent the examination sites.

mass and minimize over-penetration [4]. Another technique recommended by the WHO is to stretch the skin flat and inject the needle at a $90^{\circ}$ angle [5]. A randomized control study was previously performed to assess adverse local reactions and immunogenicity in young infants aged two - four months immunized with DPT/IPV, Hib, or meningococcal vaccines using a 16- or 25-mm needle. The incidence of local adverse reactions was lower in those immunized using the 25-mm needle with similar immune responses [6]. IM has been recommended for the vaccination of young infants with the recommended site shifting from the thigh $(<12$ months of age $)$ to the deltoid muscle (1 - 1.5 year) [4] [5]. Severe pain was reported in 30.5\% of children (18 months) injected with DPTIPV in the thigh, but in only $8.1 \%$ of those injected in the deltoid muscle [7]. Children aged 18 months vaccinated with DPT/IPV in the thigh using a 16- or 25-mm needles exhibited decreased movement of the extremity significantly more often than those injected in the arm (49.9\% vs. 25.6\%). Redness and swelling were more frequent after an injection in the arm than in the thigh and were common in groups injected in the thigh with a 16-mm needle [7]. Petousi-Harris reviewed vaccine injection techniques for sites, needle gauge, needle length, 
and angle of the injection. The WHO and CDC recommend the thigh for young infants and deltoid muscle for older children at a $90^{\circ}$ angle using 23 - 25-gauge needles that are $25 \mathrm{~mm}$ in length [4] [5]. A lower incidence of severe local adverse reactions was noted using longer needles, without any significant differences in the incidence of systemic adverse events [8]. Similar findings of a lower incidence of local adverse events using longer needles have been reported in several studies [9].

Needle length was previously evaluated in a clinical study to assess the lower incidence of severe adverse events following immunization using needles 5/8 inch $(16 \mathrm{~mm})$ or 1 inch $(25 \mathrm{~mm})$ in length [6]-[9]. Longer needles have been recommended because of the lower incidence of redness and swelling. However, a suitable needle length needs to be determined based on anatomical examinations.

Several studies have been conducted in order to assess appropriate needle lengths using ultrasonic echograms. Cook and Murtagh examined subcutaneous tissue and muscle thickness in two-, four-, six-, and 18-months-of age infants. The mean subcutaneous tissue thickness was $8.6 \mathrm{~mm}$ at two months, $9.4 \mathrm{~mm}$ at four months, 10.2 $\mathrm{mm}$ at six months, and $8.1 \mathrm{~mm}$ at 18 months. Muscle layer thickness was $10.5 \mathrm{~mm}$ at two months, $12.2 \mathrm{~mm}$ at four months, $14.8 \mathrm{~mm}$ at six months, and $16.5 \mathrm{~mm}$ at 18 months. A $16-\mathrm{mm}$ needle was suitable for IM at the anterolateral thigh when injected at a $90^{\circ}$ angle and a $25-\mathrm{mm}$ needle when injected at a $45^{\circ}$ angle into the skin's surface [10] [11]. Lippert and Wall reported that injections using 1- and 1-1/4-inch needles into the thigh resulted in 11\% (11 out of 100) and 39\% (34 out of 88) of cases being over-penetrated, respectively, using MRI or CT. In the deltoid site, 1-inch needles may result in a higher incidence of over-penetration [12]. They recommended the revision of needle lengths in order to minimize the risk of over-penetration. According to the results of the present study, the optimal needle length for IM appears to be $5 / 8$ inch injected at a $90^{\circ}$ angle into the skin surface for all infants irrespective of the injection site at the thigh or deltoid muscle, with stretching the skin flat. No racial differences appear to exist in appropriate needle lengths for IM. Limitation of the study is that the data is based on the anatomical examination, and the situation would be changeable in clinical settings.

\section{Acknowledgements}

The authors thank the doctors, nurses, radiological technician, parents, and children involved in the conduct of this study. We would also like to thank Yukikazu Hayashi and Ryo Watanabe in A2 Healthcare Corporation for their assistance in statistical analysis. The study fund sponsor was Japan Vaccine Co., Ltd.

\section{References}

[1] Japan Pediatric Society Committee on Muscular Contracture (1983) A Report of Muscular Contracture. Journal of Japan Pediatric Society, 87, 1067-1099 (in Japanese).

[2] Herzog, C. (2014) Influence of Parenteral Administration Routes and Additional Factors on Vaccine Safety and Immunogenicity: A Review of Recent Literature. Expert Reviews of Vaccines, 13, 399-415. http://dx.doi.org/10.1586/14760584.2014.883285

[3] Kashiwagi, Y., Maeda, M., Kawashima, H. and Nakayama, T. (2014) Inflammatory Responses Following Intramuscular and Subcutaneous Immunization with Aluminum-Adjuvanted or Non-Adjuvanted Vaccines. Vaccine, 32, 33933401. http://dx.doi.org/10.1016/j.vaccine.2014.04.018

[4] Centers for Disease Control and Prevention (2011) General Recommendations on Immunization-Recommendations of the Advisory Committee on Immunization Practices (ACIP). Morbidity and Mortality Weekly Report (MMWR), 260, $1-60$.

[5] WHO (2015) Immunisation in Practice. A Practical Resource Guide for Healthcare Workers. World Health Organisation, Geneva.

[6] Diggle, L., Deeks J.J. and Pollard, A.J. (2006) Effect of Needle Size on Immunogenicity and Reactogenicity of Vaccines in Infants: Randomised Controlled Trial. British Medical Journal, 333, 571. http://dx.doi.org/10.1136/bmj.38906.704549.7C

[7] Ipp, M.M., Gold, R., Goldbach, M., et al. (1989) Adverse Reactions to Diphtheria, Tetanus, Pertussis-Polio Vaccination at 18 Months of Age: Effect of Injection Site and Needle Length. Pediatrics, 83, 679-682.

[8] Petousis-Harris, H. (2008) Vaccine Injection Technique and Reactogenicity-Evidence for Practice. Vaccine, 26, 6299-6304. http://dx.doi.org/10.1016/j.vaccine.2008.08.052

[9] Beirne, P.V., Hennessy, S., Cadogan, S., Shiely F., Fitzgerald, T. and MacLeod, F. (2015) Needle Size for Vaccination Procedures in Children and Adolescents. The Cochrane Collaboration, The Cochrane Library. http://dx.doi.org/10.1002/14651858.CD010720.pub2 
[10] Hick, J.F., Charboneau, J.W., Brakke, D.M. and Goergen, B. (1989) Optimum Needle Length for Diphtheria-TetanusPertussis Inoculation of Infants. Pediatrics, 84, 136-137.

[11] Cook, I.F. and Murtagh, J. (2002) Needle Length Required for Intramuscular Vaccination of Infants and Toddlers. An Ultrasonographic Study. Australian Family Physician, 31, 295-297.

[12] Lippert, W.C. and Wall, E.J. (2008) Optimal Intramuscular Needle-Penetration Depth. Pediatrics, 122, e556-563. http://dx.doi.org/10.1542/peds.2008-0374 